\title{
GRaTIS: Sensing and Intelligence for Performance in The Presence of Legacy Networks
}

\author{
Dola Saha, Aveek Dutta, Dirk Grunwald and Douglas Sicker \\ University of Colorado, Boulder, CO 80309-0430 USA \\ Email: \{Dola.Saha, Aveek.Dutta, Dirk.Grunwald, Douglas.Sicker\}@ colorado.edu
}

\begin{abstract}
Recent work has examined techniques to estimate the "best" modulation rate for data networks such as OFDMbased $802.11 a / g$. Each rate is effective in a range of signal-tonoise ratios (SNRs) but the limited number of practical rates often force the transmitter to "step down" to a lower data rate despite having higher SNR to the receiver.

In this paper we describe, evaluate and implement a practical multiuser communication scheme that transmits two packets in the time normally needed to transmit a single packet, increasing aggregate throughput precisely when it is most needed - when the network is busy and suffers from rate unfairness.

Because the method transmits a group of packets simultaneously, we call this scheme Group Rate Transmission with Intertwined Symbols, or GRaTIS. This method uses SNR sensing and knowledge about the network to achieve improved performance in the presence of legacy network members.
\end{abstract}

\section{INTRODUCTION}

Modern wired, wireless and optical communication systems use different modulation schemes to balance data rates against error rates. These transmission rates are robust under varying SNRs. For example, the QPSK-3/4 rate requires an SNR of $8.0 \mathrm{~dB}$ to deliver $18 \mathrm{Mb} / \mathrm{s}$ throughput. The $16 \mathrm{QAM}-1 / 2$ rate, delivering $24 \mathrm{Mb} / \mathrm{s}$, could be used if the SNR was $12.5 \mathrm{~dB}$, however if the SNR falls between $8 \mathrm{~dB}$ and $12.5 \mathrm{~dB}$ we get a "better" signal that reduces packet drops but results in little net throughput improvement. In this paper, we show that it is possible to exploit the higher SNR of this primary node to encode another message for a second receiver, increasing the aggregate network bandwidth. In doing so we introduce multiple data rates to provide an even gradation of SNR across a group of receivers - we call this Group Rate Transmission with Intertwined Symbols, or GRaTIS.

Our method depends on SNR diversity between the receivers. Fortunately, most networks exhibit considerable SNR diversity. Figures 1(a) and 1(b) show the distributions of SNR at a number of locations measured at a SIGCOMM conference in 2008 and measured by us around a university campus respectively. Figure 1(b) also shows a SNR profile that is typical to a home network shared by two users with high volume video streaming. The diversity in SNR occurs because of the spatial layout of nodes, room geometries and interference from other sources.

Our implementation is based on OFDM-based 802.11a/g Physical(PHY) Layer, but can be easily extended to any OFDM-based technologies, like WiMax, LTE, DVB, etc. The benefits of GRaTIS are the larger number of group

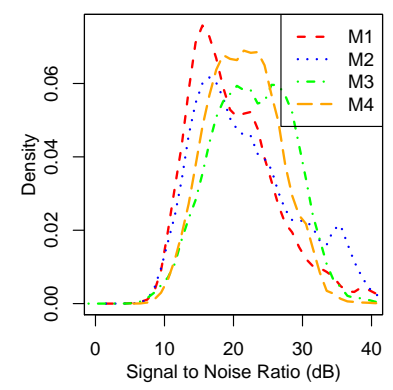

(a) SNR Profile 1

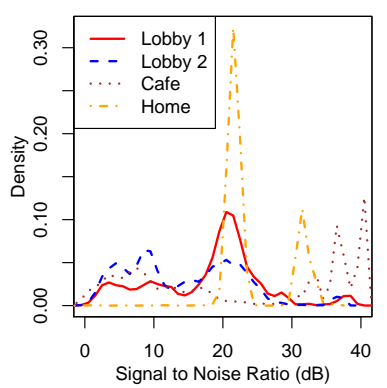

(b) SNR Profile 2
Fig. 1. Variation of SNR due to spatial diversity in 802.11a/g networks. Profile 1: Measured indoors by 4 packet sniffers at SIGCOMM 2008 [1] Profile 2: Measured indoors in common areas around a university cafe and lobbies and also in home networks.

rates, which provide increased opportunities for improving performance but doesn't impact performance when not used. GRaTIS can extend legacy networks using knowledge of the network about the capabilities (either observed or stated) of existing nodes. It is easily implemented on conventional wireless signal processing pipelines and, it complements the gains with other physical layer techniques. Using different analysis techniques we show that this scheme is both practical, profitable and implementable.

\section{GRATIS: FREE BITS}

In this section, we describe GRaTIS, which identifies two "layers" within the standard constellations available in $802.11 \mathrm{a} / \mathrm{g}$. These two layers are used to map the packets of two different users to form one single packet, such that the time required to transmit the merged packet in GRaTIS is less than the time required to transmit two separate packets in the best achievable data rate of a legacy system such as $802.11 \mathrm{a} / \mathrm{g}$. The two layers are designed in such a way that Base Layer be decoded by a legacy decoder. The second layer is obtained by extracting a few bits after the legacy demodulation system converts the I/Q samples from the analog domain to the binary domain, and we term it as the GRaTIS Layer. The second packet is transmitted during the transmission of the first packet, without any extra airtime, and comes as free bits to the (non-legacy) receiver with a higher SNR - those free bits increase the aggregate throughput of the network.

For example, assume the SNR of two nodes $n_{1}$ and $n_{2}$ are $S N R_{n_{1}}$ and $S N R_{n_{2}}$ respectively. Also, there exists a GRaTIS rate, where the SNR requirement for Base and GRaTIS layers are $S N R_{b}$ and $S N R_{g}$ respectively, and $S N R_{b}<=S N R_{n_{1}}$ 


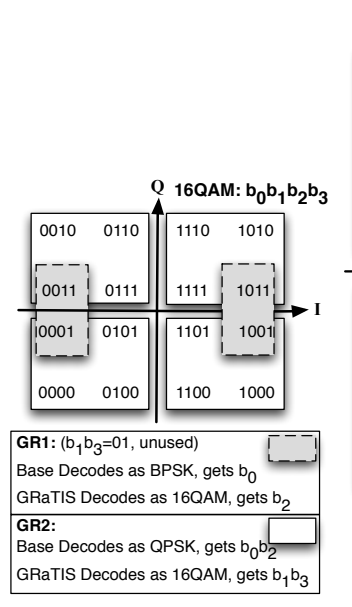

(a) GR1 and GR2

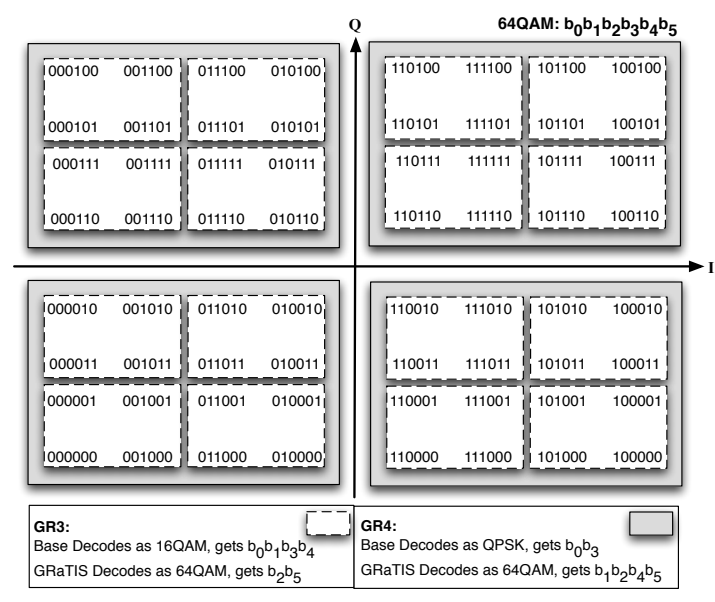

(b) GR3 and GR4

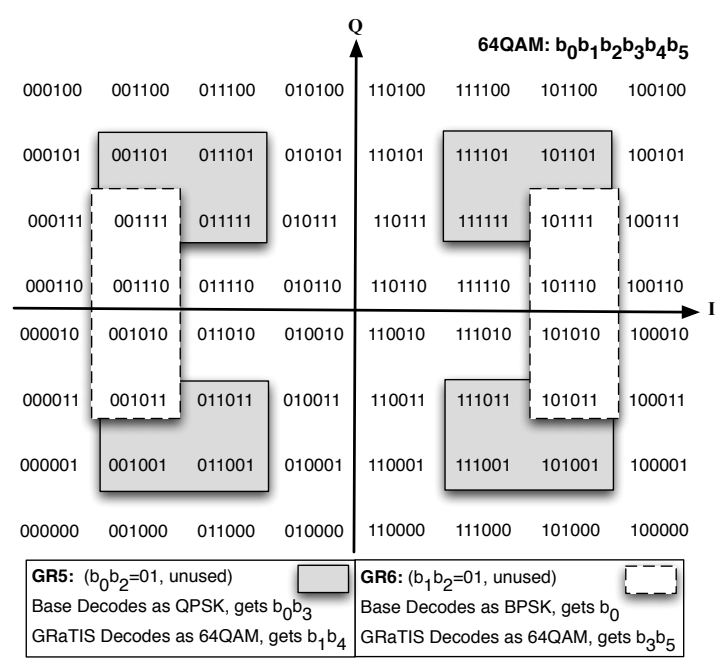

(c) GR5 and GR6

Fig. 2. Encoding and decoding of GRaTIS derived from standard 802.11a/g constellations. Rectangular regions show the transmitted cluster and the corresponding decision boundary for the base layer.

and $S N R_{g}<=S N R_{n_{2}}$. Consider the best achievable data rate in $802.11 \mathrm{a} / \mathrm{g}$ are $R_{n_{1}}$ and $R_{n_{2}}$, while that using GRaTIS are $R_{b}$ and $R_{g}$ respectively. If we consider, that there is a complete overlap of the two packets, then using GRaTIS, $n_{1}$ will transmit $x$ bytes of data using rate $R_{b}$ in time $t_{g}$ (total transmission time using GRaTIS). Since the GRaTIS layer is transmitted at the same time over the Base layer, there is no extra time required to transmit the GRaTIS layer. So, it also transmits $y$ bytes of data using rate $R_{g}$ in time $t_{g}$, giving a total data rate of $\frac{(x+y)}{t_{g}}$. The achievable data rate in $802.11 \mathrm{a} / \mathrm{g}$ for the two transmissions will be $\frac{(x+y)}{t_{1}+t_{2}}$, while using GRaTIS it will be $\frac{(x+y)}{t_{g}}$. The pair of rates $R_{b}$ and $R_{g}$ are selected as one of the GRaTIS rates, iff $\frac{(x+y)}{t_{1}+t_{2}}<\frac{(x+y)}{t_{g}}$. Or in other words, GRaTIS rates are selected only if sensing indicates there is potential gain in throughput.

\section{A. Encoding Packets using GRaTIS}

We introduce six distinct GRaTIS rates, or methods of combining packets, as shown in figure 2 , to increase aggregate throughput of the network. The GRaTIS rates are termed GRI through GR6. We select the combination of layers as one of the GRaTIS rates if the data rate achievable by merging is more than that of two packets transmitted separately. At the transmitter, two packets are encoded independently up to the modulation subsystem as shown in figure $3(\mathrm{a})$. Then the bits of two packets, $b_{b}$ and $b_{g}$, are encoded at rates $R_{b}$ for the base and $R_{g}$ for the GRaTIS layer respectively, are combined to form a compound symbol that represents one of the constellation points corresponding to a standard modulation in $802.11 \mathrm{a} / \mathrm{g}$, denoted by $R_{m}$. This mapping ensures that the compound symbols are mapped only to the I/Q vectors that are part of a selected GRaTIS cluster. In this way, the modulator remains unchanged, as it is fed with the compound bitstream $\left(b_{m}\right)$, and modulation type $\left(R_{m}\right)$ to which it modulates. Since all the packet merging is done at the bit-level it does not require any change in the signal processing pipeline.
The clusters are selected to optimize properties of the I/Q-plane mapping used to represent information in wireless networks. For example, Figure 2(a) shows the constellation points used in GRl and GR2, which are derived from a 16QAM constellation. In $G R 1$, resultant cluster points are modulated to carry two bits of useful information, one bit for each layer. To reduce the probability of error in the base layer, the points are chosen such that the vectors in the I-plane are greater than that of BPSK mapping while the deviations in the vectors of the Q-plane is used to carry another BPSK packet in the GRaTIS layer. Out of the 4 bits available for every constellation point in the cluster, bit $b_{0}$ is used to encode the base layer, and bit $b_{2}$ contains the GRaTIS layer. The other two bits, $b_{1}$ and $b_{3}$, remain constant at 0 and 1 , respectively to map the compound symbol to the desired cluster. The shaded region shows the transmitted constellation for GR1. GR2 uses all the constellation points of a 16QAM constellation, and provides 2 bits of information per subcarrier, as is done in QPSK, to each of the two nodes. Bits $b_{0} b_{2}$ and bits $b_{1} b_{3}$ are used to encode the information of base layer and GRaTIS, respectively. As a result of such mapping when a cluster point that is closest to an axis crosses the axes due to channel noise, a symbol error occurs for the base layer, as seen in QPSK modulations. However, no error is incurred in the GRaTIS layer. Hence, this type of mapping provides some extra error protection to the GRaTIS layer.

GR3 and GR4 utilizes 64QAM constellation to encode the two layers as shown in figure 2(b). GR3 provides 16QAM data rate to the base layer using bits $b_{0} b_{1} b_{3} b_{4}$, while $G R 4$ provides a QPSK data rate to the base layer using bits $b_{0} b_{3}$. The remaining bits are used to encode the data of GRaTIS layer. GR5 and GR6 uses a cluster derived from a 64QAM constellation as shown in figure 2(c). GR5 uses bits $b_{0} b_{3}$ for the base layer and bits $b_{1} b_{4}$ for the GRaTIS, providing QPSK data rate to both the packets. Bits $b_{2} b_{5}$ are modulated as 1 , to generate the desired cluster as shown in dash-dotted lines. GR6 uses bits $b_{0}$ and $b_{3} b_{5}$ to encode the information of the 


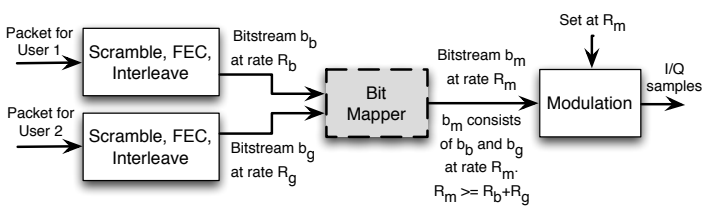

(a) Transmitter Pipeline

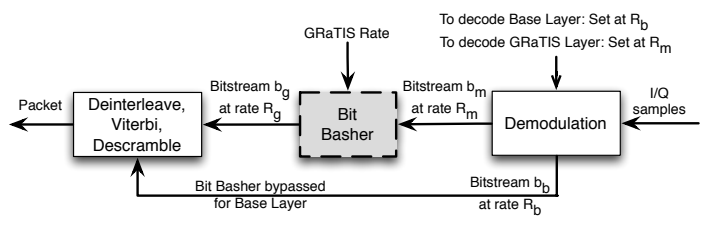

(b) Receiver Pipeline

Fig. 3. Transceiver pipeline for GRaTIS - shaded subsystems show additional nrmescese remired for $\mathrm{AR}$ aTIS

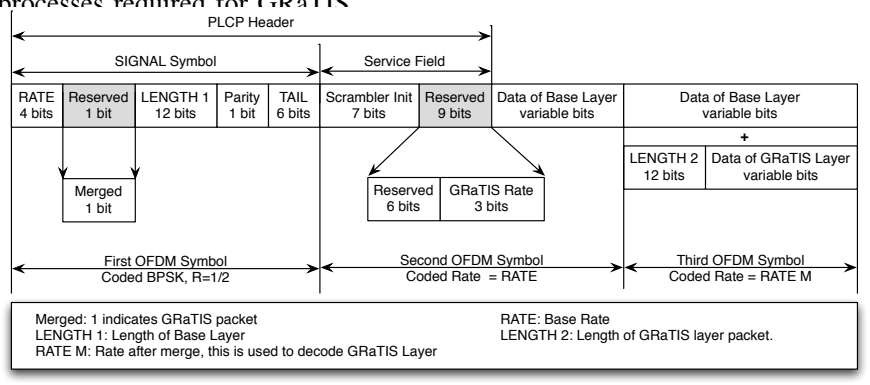

Fig. 4. Modified 802.11a/g PLCP header - shaded fields indicate modifications to support GRaTIS.

base and GRaTIS layers, respectively, while modulating bits $b_{1}$ as 0 and $b_{2}$ as 1 leading to the desired cluster points.

\section{B. Decoding Packets using GRaTIS}

GRaTIS has two layers, intended for two receivers. The base layer is encoded in such a way that its decoding is the same as decoding any generic $802.11 \mathrm{a} / \mathrm{g}$ packet. For example, in GR1, bit $b_{0}$ of 16QAM modulation is encoded as the base layer. So, the constellation points generated in the left side of the Q-plane always yield a value of 0 , and the right side of the Q-plane is always decoded as a 1. This phenomenon is the same as BPSK modulation. Thus, at the receiver side, the base layer of GRI can be simply decoded as a BPSK packet.

Figure 3(b) shows the demodulation pipeline for a GRaTIS compatible node. The GRaTIS layer is first decoded as the constellation from which the GRaTIS cluster has been derived $\left(R_{m}\right)$. Then, the bits designated for the GRaTIS layer are extracted using the GRaTIS rate information, to form the bit stream for the second packet $\left(b_{g}\right)$ at rate $R_{g}$. The rest of the receiver decode pipe remains unchanged.

\section{Medium Access Control for GRaTIS}

To successfully decode a GRaTIS packet, a node needs to know the encoding information of the packet. We use the reserved bits of the PLCP Header of IEEE 802.11a/g packet to provide the encoding information as shown in figure 4 .

In the $802.11 \mathrm{a} / \mathrm{g}$ PHY layer, each message must be individually acknowledged. One major hindrance in using multiuser communications such as GRaTIS is the need for those acknowledgments. For this we rely on a simultaneous acknowl- edge mechanism (SMACK) [2] to gather acknowledgments from multiple recipients of the merged packet. SMACK will reduce the overhead of scheduling multiple acknowledgment packets and will reduce the multi-party acknowledgment time.

\section{GRATIS: RATE ANALYSIS}

In this section we evaluate the bit error rate (BER) and packet error rate (PER) for various group rates in GRaTIS, in the presence of Additive White Gaussian Noise (AWGN) using the standard techniques used to analyze digital communication performance. At the receiver, the noisy constellation points in the I/Q plane are mapped to corresponding bits by using maximum likelihood (ML) decoding. Constellation points are required to be within an area in the IQ-plane defined by a modulation dependent decision boundary to ensure error free decoding. The BER for such a scheme is given by eq. 1 .

$$
P_{B}(E)=\frac{1}{2} \operatorname{erfc}\left(\sqrt{\frac{\Delta_{E}(i, j)}{4 N_{0}}}\right)
$$

The bit error rate for an arbitrary modulation scheme and ML decoding boundaries is upper bounded by:

$$
\begin{aligned}
P_{W U B}(E) & =\sum_{j=1}^{M-1} \sum_{i \neq j} \frac{1}{2 M} \operatorname{erfc}\left(\sqrt{\frac{\Delta_{E}(i, j)}{4 N_{0}}}\right) \\
& =\sum_{k=1}^{N} \frac{A_{d}(k)}{2 M} \operatorname{erfc}\left(\sqrt{\frac{\Delta_{E}(k)}{4 N_{0}}}\right)
\end{aligned}
$$

where,

- $\Delta_{E}(i, j)=$ Squared Euclidean distance between two distinct constellation points $i$ and $j$.

$-N=$ Possible different squared Euclidean distances in the decoded constellation, where $N \leq M(M-1) / 2$.

$-M=$ Total number of decoded constellation points.

$-N_{0}=$ Additive white noise power.

- $\Delta_{E}(k)=$ Distinct pairwise Euclidean distance in the decoded constellation.

- $A_{d}(k)=$ Number of signal pairs having squared Euclidean distance of $\Delta_{E}(k)$.

Using eq. 2 we can compute the BER for any constellation and ML decision boundary. The detailed calculation of BER is not included due to space constraints. The PER for a packet size of 128 bytes after Viterbi decoding is computed using the maximum free distance for a particular coding rate and its corresponding distance spectrum.

In the BER computation we consider that the GRaTIS constellations are mapped using Gray code [3] and encoded using a $1 / 2$ rate (except for 64QAM, which is encoded using a $2 / 3$ rate) as well as $3 / 4$ rate convolution code. This analysis has been done to ascertain the operating range of different group rates and their potential benefits when used to merge packets using GRaTIS. The BER computation for the GRaTIS layer is similar to that of the base layer and can be calculated using eq. 2. We discuss the performance of both the layers using a testbed in $\S \mathrm{V}$. The theoretical and testbed results are listed 
TABLE I

THROUGHPUT AND SNR REQUIREMENTS FOR 802.11A/G AND GRATIS RATES

\begin{tabular}{|c|c|c|c|c|c|c|c|}
\hline \multirow{3}{*}{ Mod } & \multirow{3}{*}{$\mathrm{CR}^{1}$} & \multirow{2}{*}{\multicolumn{2}{|c|}{$\begin{array}{c}\text { Data Rate } \\
(\mathrm{Mbps})\end{array}$}} & \multicolumn{4}{|c|}{$\mathrm{SNR}(\mathrm{dB})$} \\
\hline & & & & \multicolumn{2}{|c|}{ Base } & \multicolumn{2}{|c|}{ GRaTIS } \\
\hline & & Link & Grp & $\mathrm{Th}^{2}$ & Exp & $\mathrm{Th}^{2}$ & Exp \\
\hline$\overline{\overline{\text { BPSK }}}$ & $\overline{1 / 2}$ & $\overline{\overline{6}}$ & \multirow{8}{*}{$n / a^{3}$} & 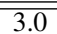 & $\overline{4.5}$ & \multirow{8}{*}{$n / a^{3}$} & \multirow{8}{*}{$\mathrm{n} / \mathrm{a}^{3}$} \\
\hline BPSK & $3 / 4$ & 9 & & 5.0 & 6.0 & & \\
\hline QPSK & $1 / 2$ & 12 & & 6.0 & 7.0 & & \\
\hline QPSK & $3 / 4$ & 18 & & 8.0 & 9.0 & & \\
\hline 16QAM & $1 / 2$ & 24 & & 12.5 & 13.0 & & \\
\hline 16QAM & $3 / 4$ & 36 & & 17.0 & 18.0 & & \\
\hline 64QAM & $2 / 3$ & 48 & & 19.5 & 24.0 & & \\
\hline 64QAM & $3 / 4$ & 54 & & 21.0 & 26.0 & & \\
\hline GR1 & $1 / 2$ & 6 & 12 & 3.5 & 5.0 & 17.0 & 17.0 \\
\hline GR6 & $1 / 2$ & 6 & 18 & 4.5 & 5.0 & 18.5 & 21.0 \\
\hline GR5 & $1 / 2$ & 12 & 24 & 7.5 & 7.5 & 18.5 & 23.0 \\
\hline GR2 & $1 / 2$ & 12 & 24 & 9.0 & 10.5 & 11.5 & 15.0 \\
\hline GR4 & $1 / 2$ & 12 & 36 & 15.0 & 15.0 & 19.0 & 23.0 \\
\hline GR3 & $1 / 2$ & 24 & 36 & 17.0 & 16.5 & 20.0 & 25.0 \\
\hline GR1 & $3 / 4$ & 9 & 18 & 5.5 & \multirow{6}{*}{$\mathrm{n} / \mathrm{i}^{4}$} & 19.5 & \multirow{6}{*}{$\mathrm{n} / \mathrm{i}^{4}$} \\
\hline GR6 & $3 / 4$ & 9 & 27 & 7.0 & & 20.5 & \\
\hline GR5 & $3 / 4$ & 18 & 36 & 10.0 & & 20.5 & \\
\hline GR2 & $3 / 4$ & 18 & 36 & 13.0 & & 14.5 & \\
\hline GR4 & $3 / 4$ & 18 & 54 & 19.5 & & 21.5 & \\
\hline GR3 & $3 / 4$ & 36 & 54 & 20.0 & & 22.0 & \\
\hline
\end{tabular}

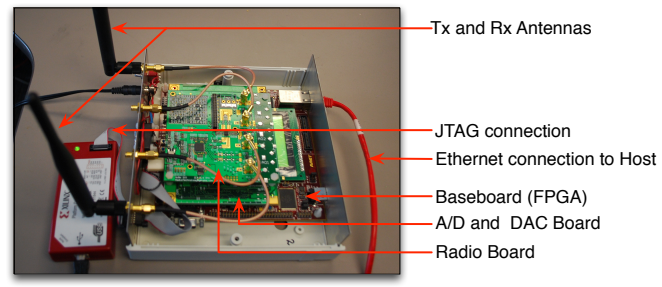

Fig. 5. SDR platform used to implement GRaTIS.

in Table I which shows the SNR requirements for a $2 \%$ PER along with the SNR requirement for the $802.11 \mathrm{a} / \mathrm{g}$ standard rates. The SNR - throughput relationship for various group rates are used when downlink packets are being considered to be merged. As shown in Table I, the group rates provide a variety of step-down rates while utilizing SNR diversity in the network to increase the aggregate throughput of the network.

\section{IMPLEMENTING GRATIS}

Most of encoding of GRaTIS can be done in software. However, access to the packetization engine of the MAC layer is required. Since, this abstraction layer is not available to us from commodity hardware, we implemented this technique using a hybrid software defined radio (SDR) based on a Virtex5 FPGA (Figure 5) that can transmit and receive generic 802.11a/g data packets [3]. This prototype is based on previous work [4], [5], and gives access to the packetization layer of the MAC where the data bits from two users are combined to form a symbol in the I/Q plane. This is accomplished by the bit mapper unit shown in 3(a).

The base layer receiver can be oblivious of any enhanced layer. Depending on the GRaTIS rate used to encode the second packet, the demodulator extracts the additional bits: typically termed as bit slicing or bit bashing. Figure 3(b) shows the basic structure of the modified demodulator. The bit

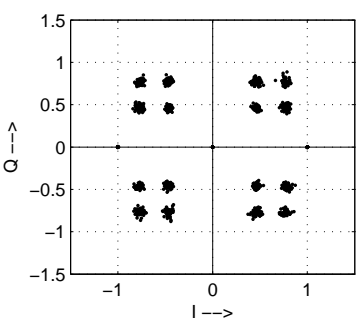

(a) GR5

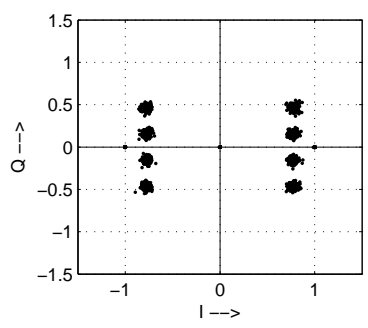

(b) GR6
Fig. 6. Example GRaTIS constellations transmitted using the SDR prototype.

basher unit is responsible for extracting the bits of the GRaTIS layer. Other receiver subsystems prior to the demodulator, e.g., the synchronizer and the equalizer, remain unchanged for implementing GRaTIS. Figure 6 shows constellations of GR5 and GR6 produced by our prototype SDR platform.

The receiver uses standard OFDM demodulation techniques and largely the same MAC layer. Although the hardware chain is unchanged, the software controlling the binary operations that follow the demodulator are updated; this is usually a software upgrade. This is a distinct advantage over other multiuser decoding techniques that rely heavily on complex signal processing algorithms, involving custom constellations and more general control of the I-Q mapping that prevents operation in legacy networks. GRaTIS provides an example of how sensing and software radios can harness the power of existing resources while improving network performance.

\section{GRATIS: PutTING IT TO WORK}

A testbed has been setup with three nodes, one transmitter and two receivers in an indoor laboratory environment. The nodes are placed at a distance of approximately $5 \mathrm{~m}$ on work desks in enclosed cubicles. Channel quality and SNR variation is obtained by controlling the transmit power as well the location of the receiver nodes relative to the transmitter. The average throughput and average SNR required for $2 \%$ PER across different node arrangements have been measured. The group rates proposed in $\S \mathrm{II}$, with $1 / 2$ rate convolution coding for both the layers have been compared to the standard rates available in $802.11 \mathrm{a} / \mathrm{g}$. SNR is measured as the ratio of the signal power (average of 5 OFDM symbols after a packet is detected) and noise power (average of 5 OFDM symbol after the end of packet).

Figure 7 shows the performance of the base and GRaTIS layer of six group rates, along with standard modulations, BPSK, QPSK, 16QAM and 64QAM. For each modulation, we plot the physical layer throughput and mark the minimum SNR required for a PER of $2 \%$, below which it cannot be used reliably. The base and GRaTIS layers are denoted by suffixes '(b)' and '(g)' respectively.

As in figure 7, GRl(b) provides BPSK rate, and have an SNR requirement between BPSK and QPSK. Thus, when a node becomes unreachable in QPSK, we can use GRl to combine packets, and send a different packet to another node reachable at higher SNR (17dB or higher) using GRl $(g)$. Similarly, for other GRaTIS rates, there is an oppportunity to 


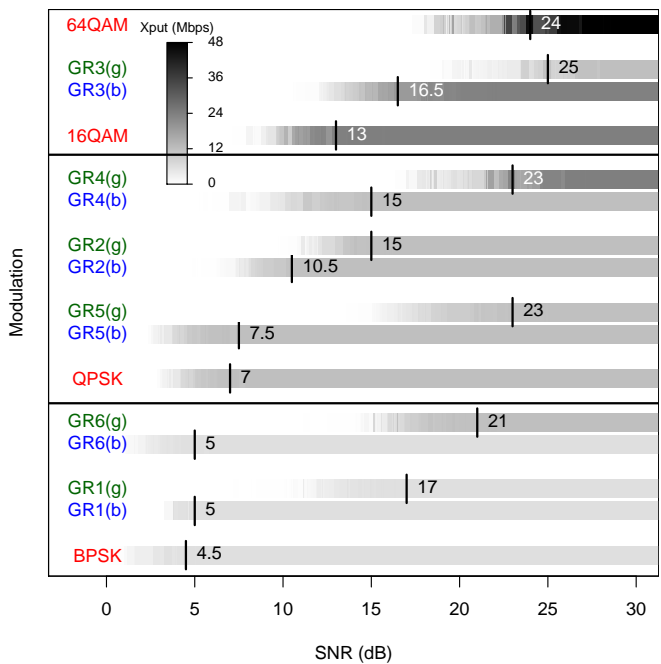

Fig. 7. Over-the-air link throughput of GRaTIS((b)-Base, (g)-GRaTIS) and $802.11 \mathrm{a} / \mathrm{g}$ rates with increasing SNR. The rates are grouped according to the increasing base rate. The numbers against each rate denote the SNR required to decode a 128 byte, $1 / 2$ rate convolution coded packet with $2 \%$ PER.

send extra packet to another node while transmitting a packet to one. Just an exception to the other combinations, GR4(b) provides throughput equal to that of QPSK, but requires more SNR than 16QAM to be decoded correctly. It might seem that this group rate does not provide the best effort rate to the base layer. Careful observations reveals that $G R 4(g)$ provides a throughput equal to that of 16QAM, and has a lower SNR requirement than 64QAM. So, we can transmit a packet in $G R 4(g)$ to a node when its SNR falls below 64QAM, and $G R 4(b)$ then can be used to transmit any extra bits as a GRaTIS layer.

\section{GRATIS: Practical Gains}

Theoretical and experimental results in $\S I I I$ and $\S \mathrm{V}$ respectively show the potential benefits of using GRaTIS in modern networks like $802.11 \mathrm{a} / \mathrm{g}$. It is sometimes difficult to understand the benefits of a particular wireless optimization from PER plots and bench experiments. Also, testbed implementations, where the network is flooded with UDP packets of the same packet length do not represent a realistic scenario of wireless networks. We want to know - a) How often do stations have sufficient SNR diversity to exploit GRaTIS? b) Does GRaTIS provide gains with variable packet lengths of the users? c) How useful is the combined coding efficiency over $802.11 \mathrm{a} / \mathrm{g}$ network? d) Does GRaTIS work in different scenarios, like a conference hall, university cafeteria or home network? To determine the system benefits of GRaTIS, we analyzed captured packet traces from SIGCOMM 2008 dataset [1]. The SIGCOMM traces reported signal and noise power in the Prism header [6], which have been converted to report SNR in $\mathrm{dB}$.

We analyze the traces based on the monitor's view of the network, merging downlink data packets based on the SNRs received from the clients. Two downlink data packets are merged only if the time required to transmit a merged GRaTIS packet is less than the time required to transmit two individual packets using $802.11 \mathrm{a} / \mathrm{g}$. The queue length is finite, and only
10 packets have been considered to be available at any time for merging. Off-the-shelf wireless AP queue capacity varies from 39 to 337 packets [7], which indicates that our projected performance might improve in real networks. We used the experimental results, as reported in Table I, to compute the airtime usage in both of the cases. We consider all coding rates for $802.11 \mathrm{a} / \mathrm{g}$, but do not consider $3 / 4$ coding rate for GRaTIS. With both the coding rates available for GRaTIS, there will be more opportunities to merge packets, for example merging a packet of $3 / 4$ coding rate with another of $1 / 2$ coding rate. This is a conservative approach to show the improvement of using GRaTIS over $802.11 \mathrm{a} / \mathrm{g}$, but will give a lower bound on the possible gains.

We selected one random monitor on one of the days at SIGCOMM, Monitor 4 on Aug-19, and compute the throughput gain achieved per min using our method. Figure 8 shows the temporal variation of the percentage gain/min in throughput if GRaTIS is used. The volume of downlink data packets transmitted per minute, and the percentage of packets merged per minute is also shown. A simple moving average (SMA) of 10 data points shows that the average gain goes up to $80 \%$, with instantaneous gain/min. goes up to $90 \%$ over $802.11 \mathrm{a} / \mathrm{g}$ network. The gain is proportional to the percentage of packets being merged. Most of the time, when there is network traffic, GRaTIS could merge $\approx 70 \%$ of the packets. We notice that the volume of packets drop significantly at time $100 \mathrm{mins}$, which we believe is the lunch hour. Most of the packets observed by the monitor have very low SNR during that lunch period, and GRaTIS had little opportunity to combine packets due to limited diversity in client SNR and the low number of packets sent. To ensure that we receive similar gains in other days using the trace from other monitors as well, we computed average gain in each day for each monitor. Results show consistent gains in other scenarios as well.

We have also captured packet traces in the common areas of our university with more than 50 active users, and in a home network of two users, the SNR variation of which is shown in 1(b). We omit the results of these captured packet analysis due to space constraints, but results show consistent gains in all the scenarios with maximum gain in throughput per min going up to $120 \%$.

This analysis shows that when the clients SNR vary in a diverse range, GRaTIS can be used to combine packets and gain airtime, which can essentially be used to transmit more packets and increase the overall throughput of the network.

\section{RELATED WORK}

Packet merging techniques exist in forms of Hierarchical Modulation, Superposition Coding and Network Coding. Hierarchical Modulation [8] is used to transmit the same information to multiple users in digital video broadcasts. GRaTIS can transmit completely different information to multiple users.

Other packet mixing techniques in wireless networks either employ superposition coding [9] or network coding [10] or a combination of the two [11]. Superposition coding relies on iterative decoding by decoding the base layer first and then 


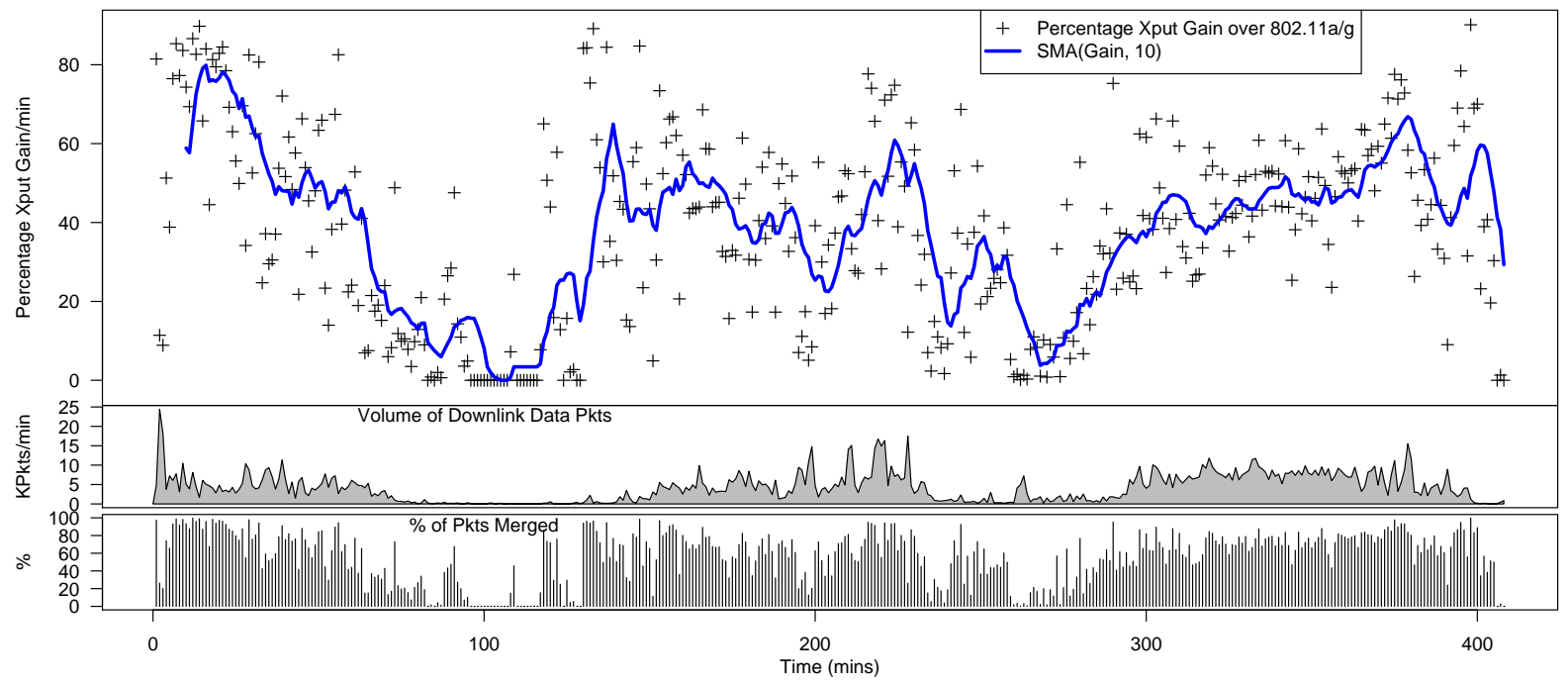

Fig. 8. An example implementation of GRaTIS when applied on the downlink data packets in an infrastructure 802.11a/g network with $>50$ users. The first plot shows the percentage gain in aggregate throughput per minute with GRaTIS. A 10 minute Simple Moving Average (SMA) of the gain in also shown. The second plot shows the volume of packets in Kpackets/min while the third plot shows the percentage of packets transmitted per minute using GRaTIS.

re-modulating it to extract the higher layer, which is more complicated to implement. In comparison GRaTIS provides a simpler decoder structure and offers flexibility by providing more practical data rates within the realm of wireless networks. Also under similar operating conditions, GRaTIS provides higher network throughput compared to superposition coding by careful mapping of the bits to the I/Q plane, the comparison of which has been omitted due to space constraints.

Network coding relies on overhearing of a packet which is used to decode a second packet. However, network coding fails if the first packet is not overheard by the intended receiver. On the other hand, GRaTIS increases the applicability of network coding because the network coding message can always be used as a GRaTIS-layer message even if other messages are not queued.

In [12], authors propose mixing bits for relaying purposes, but the mapping of bits to the constellations is different from GRaTIS. The work does not consider the fact that packet mixing will increase the SNR requirements for decoding both the layers. On the contrary, our work is practical and the SNR requirement for each layer has been shown both theoretically and experimentally. The packet trace analysis with variable packet size shows GRaTIS is applicable to most common wireless network scenarios.

\section{CONCLUSION}

GRaTIS provides an efficient method of simultaneous packet transmission and reception to increase the aggregate throughput of the network. We have implemented the protocol in hardware and experimental results show several possibilities of use of GRaTIS giving unforeseen gains in throughput in wireless networks. Applying GRaTIS on real-time packet trace analysis reveals that even with a few simple combinations, we can gain significant airtime. Also through our analysis we show that GRaTIS provides better error performance than other contemporary simultaneous packet transmission techniques, making it a suitable candidate for emerging wireless networks.

\section{REFERENCES}

[1] A. Schulman, D. Levin, and N. Spring, "CRAWDAD trace set umd/sigcomm2008/pcap (v. 2009-03-02)," Downloaded from http://crawdad.cs.dartmouth.edu/umd/sigcomm2008/pcap, Mar. 2009.

[2] A. Dutta, D. Saha, D. Grunwald, and D. Sicker, "Smack: a smart acknowledgment scheme for broadcast messages in wireless networks," SIGCOMM Comput. Commun. Rev., vol. 39, no. 4, pp. 15-26, 2009.

[3] Part 11: Wireless LAN Medium Access Control (MAC) and Physical Layer (PHY) Specifications, IEEE Computer Society : LAN/MAN Standards Committee. [Online]. Available: http://standards.ieee.org/ getieee802/download/802.11-2007.pdf

[4] J. Fifield, P. Kasemir, D. Grunwald, and D. Sicker, "Experiences with a platform for frequency agile techniques," in DYSPAN, 2007.

[5] A. Dutta, J. Fifield, G. Schelle, D. Grunwald, and D. Sicker, "An intelligent physical layer for cognitive radio networks," in WICON '08: Proceedings of the 4th international conference on Wireless internet, 2008.

[6] "Madwifi driver, http://madwifi-project.org/." [Online]. Available: http: //madwifi-project.org/

[7] F. Li, M. Li, R. Lu, H. Wu, C. Mark, and K. Robert, "Measuring queue capacities of ieee 802.11 wireless access points," in Broadband Communications, Networks and Systems, 2007. BROADNETS 2007. Fourth International Conference on, sept. 2007, pp. 846 -853.

[8] K. Uz, M. Vetterli, and D. LeGall, "Interpolative multiresolution coding of advance television with compatible subchannels," Circuits and Systems for Video Technology, IEEE Transactions on, vol. 1, no. 1, pp. 86-99, Mar 1991.

[9] R. Ganti, Z. Gong, M. Haenggi, C. Lee, S. Srinivasa, D. Tisza, S. Vanka, and P. Vizi, "Implementation and experimental results of superposition coding on software radio," in Communications (ICC), 2010 IEEE International Conference on, May 2010, pp. 1 -5.

[10] S. Katti, D. Katabi, H. Balakrishnan, and M. Medard, "Symbol-level network coding for wireless mesh networks," SIGCOMM Comput. Commun. Rev., vol. 38, no. 4, pp. 401-412, 2008.

[11] C.-H. Liu and A. Arapostathis, "Joint network coding and superposition coding for multi-user information exchange in wireless relaying networks," in Global Telecommunications Conference, 2008. IEEE GLOBECOM 2008. IEEE, 30 2008-dec. 4 2008, pp. 1 -6.

[12] Z. Yang, Y. Luo, and L. Cai, "Network modulation: A new dimension to enhance wireless network performance," in INFOCOM, 2011 Proceedings IEEE, april 2011, pp. $2786-2794$. 